\title{
EXPERIMENTAL RESEARCH OF ABSORPTION PROPERTIES OF RIGID FOAM FILLED CIRCULAR SEAMLESS TUBE ENERGY ABSORBER UNDER QUASI-STATIC AXIAL LOAD
}

\author{
J. Tanaskovića, ${ }^{a,}$, F. Franklin ${ }^{\mathrm{b}}$, A. Mitrovićc ${ }^{\mathrm{c}}$, A. Dišićd \\ ${ }^{a}$ University of Belgrade, Faculty of Mechanical Engineering, Kraljice Marije 16, Belgrade, Serbia \\ ${ }^{\mathrm{b}}$ School of Engineering, Newcastle University, Newcastle upon Tyne, NE1 7RU, UK \\ ${ }^{\mathrm{c}}$ University Union "Nikola Tesla", Faculty of Information Technology and Engineering, Jurija Gagarina 149a, \\ Belgrade, Serbia \\ ${ }^{\mathrm{d}}$ University of Kragujevac, Institute for Information Technology Kragujevac, Jovana Cvijića BB, Kragujevac, Serbia \\ Corresponding author: J. Tanasković, Kraljice Marije 16 Street, 11000 Belgrade, Serbia, Phone: +381 641232854 , \\ E-mail: jtanaskovic@mas.bg.ac.rs
}

\begin{abstract}
The aim of this research work is investigations of absorption characteristics of a circular seamless tube collision absorber filled by rigid polyurethane (PU) foam under axial load. Shrinking of circular tube passing through cone bushing starts after absorber is activated at the moment of collision. The energy absorption realises in three ways: elastic-plastic deformation of the tube wall, friction between absorption elements and compression the rigid PU foam inside the tube. Using rigid PU foam, as the seamless tube filler, in the process of collision energy absorption increases absorption power in comparing to with only empty tube, as well as gives gradual increases of deformation resistance during deformation process. Experimental research was prepared and realized in the laboratory using the scaled samples. The effect of rigid PU foam, configuration of absorber filled by PU foam on the absorption power and manufacturing technology of the samples, are considered and discussed in this paper. The results indicate that the shrinking foam filled tube absorber has for about $18 \%$ bigger absorption power than the empty one. Formation of numerical model and numerical analyses of shrinking foam filled tube absorber were realized using ANSYS software package. Force vs. stroke $(\mathrm{F}(\mathrm{s}))$ diagrams obtained by tests and numerical analyses are in a good correlation which confirms formed numerical model as a suitable for further quasi-static analyses and for dimensioning the similar types of absorber.
\end{abstract}

Keywords: Experimental Research, Rail Vehicles, Rigid Polyurethane Foam, Circular Tube Absorber, Numerical

Simulations 


\section{Introduction}

The availability and reliability of the railway vehicles are crucial characteristics that make the difference between popular and undesired. Today, when the running speed of the railway vehicles exceeds $300 \mathrm{~km} / \mathrm{h}$ in commercial traffic, great emphasis is placed on active and passive safety systems, both of the railway vehicles and the infrastructure. Collision energy absorption is an important measure within passive safety of railway vehicles, and design of absorber elements is a significant engineering challenge. Passenger coaches have complex body structures, especially in the front area (zone of the end beam) and have limited space available for mounting of passive safety elements. EU railway standards $[1,2]$ define strength of the vehicle's structure, including the phases of controlled deformation of the passive safety elements, and require all new vehicles to be equipped with absorption elements. Existing vehicles can be equipped with passive safety elements during refurbishment or modernization. The conception of a collision energy absorber primarily depends on the demanded absorption power [1], then on the shape and way of deformation of the absorber as well as the mounting concept. Major role of the absorption elements during collision is passenger and goods protection, through preventing distortion of the wagon's structure [2]. Strictly managed shape and flow of deformation of absorption elements transform collision kinetic energy to potential energy and friction to heat and in this way decreases values of the forces that transmitted to the passenger and goods zone of the wagon body.

Next to high speed train collisions, most common collision problem during process of forming train at the railway stations is the higher shunting speed than allowed. In the case if the shunting speed (not often case, but it's happening) exceeds defined value of maximal $10 \mathrm{~km} / \mathrm{h}$ [2] comes the overload of the standard buffer and undesired consequences like plastic deformations of the body structure elements, breaks of equipment attachments etc. This undesired case requires excluding wagon from the traffic and a significant time for failures reparation that leads to increase of maintenance costs. With the aim to minimize reparation time of the failures, wagons should be equipped with energy absorbers behind the standard buffer (as modular assembly). In this way, modular type of absorber provides easy change of deformed absorption elements at the maintenance workshops and returning wagon to traffic in a shortest possible time.

A shrinking absorber has been studied in the past [3-6]. The force vs. stroke characteristics of this type of absorber are good, with a long phase of steady force during the absorption process. The main problem of this solution is available size of mounting space that limits change of length of the tube as a way of increasing absorption capacity.

Two methods for increasing the absorption power are shrinking-folding and shrinking-splitting. The shrinking-folding process is compact and has higher absorption power, but produces peak forces (deformation resistance) [7]. The shrinking-splitting process of deformation is better because the splitting process increases absorption power without producing peaks during the absorption process [8].

Increasing the absorption power within the existing design is possible using a filler of different density inside the tube. Readt and Wall [9] present results of experimental and theoretical investigations of empty and foam-filled circular metal tubes and impact of low density PU foam. Increase of deformation resistance is effect of changing deformation way and the possibilities of the tube wall compression. Numerical analyses of foam-filled and empty tubes, as well as a concentric tubes configuration, were presented by Goel [10]. Research showed that the use of concentric tubes (especially filled by PU foam) get the significant change of shape of deformation as well as absorbed energy. The influence of an initiator was analysed for various densities of PU foam by Rezvani and Jahan [11]. Results showed the best performance with an initiator, four rings, and rigid PU foam in all criteria. Combinations of polyurethane foam and honeycomb were described by Hussein et al. [12], who found that the best specimens were square aluminium tubes filled by PU foam and with honeycomb. Elahi et al. [13] presented new theoretical relations with the aim to estimate energy absorption behaviour of empty and composite tubes filled by PU foam. Tests showed that PU foam has significant role in energy absorption. Results obtained by FE model (quasi-static and dynamic analyses of tapered tube with rectangular cross section with casted PU foam inside their) showed that the foam density has no crucial influence on the initial peak of force; while the other parameters (absorber energy and deformation resistance) increase with rise density of foam, were presented by Mirfendereski et al. [14]. Experimental results analysed by Karagiozova et al. [15] show influence of foam strength and density to quasi-static and dynamic responses. Jafarian and Rezvani [16] showed that increasing of absorption power can be realized by bitubal structures filled by PU foam as well as the shrinking of tubes. The impacts of foam core to shape of deformation and values of crushing force by using squared aluminium columns filled by PU foam were studied by Dirgantara et al. [17]. Darvizeh et al. [18] found that circumferentially grooved thick-walled tubes filled by PU foam can absorb for about $26 \%$ higher collision energy in comparison with 
empty one. The interaction between the foam and the tube wall was studied by Yalçın and Genel [19]. Experimental results showed that using a proper foam filling has influence on absorbed energy and specific absorbed energy. Experimental results, presented by Wang et al. [20], obtained by quasi-static compression tests of empty and PU foamfilled connectors showed that polyurethane foam can significantly improve the energy absorption performances.

Considering the benefits and weaknesses of different types of energy absorber presented above, shrinking of a seamless steel tube filled with high-density polyurethane foam should be a good potential solution. This combination of materials and deformation method can increase absorption power without inducing a peak force at the beginning of shrinking the tube or further peaks during the absorption process. Foam filled shrinking absorber is suitable to be a part of standard buffer i.e. to be mounted immediately behind it in the vehicles bearing structure, Fig 1a. Also, this absorber can be a part of the shrinking anticlimber system on the electric/diesel train described by Moreno et al. [26], Fig. 1b.

The experimental results presented in this paper were obtained by testing scaled samples, and done with the aim to show impact of rigid PU foam on absorption properties of shrinking tube absorber. Numerical analyses have been realised using ANSYS Workbench. Developed numerical model was verified using experimental results. Influence of different values of wall thickness and density of PU foam were analysed by numerical simulations using developed FE model. The results (obtained by test and numerical simulations) were analysed and discussed in the paper and some conclusions were done based on them.
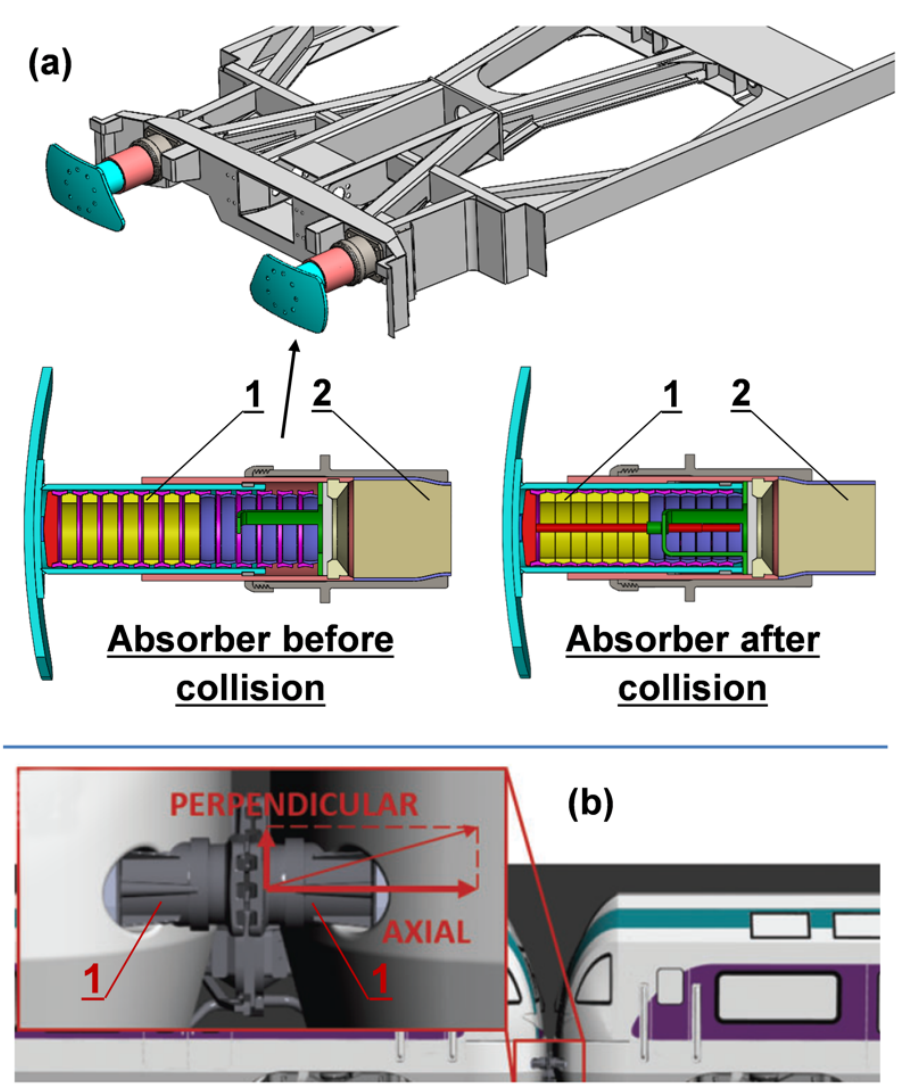

Figure 1. Possible mounting methods: (a) Absorber (Item 2) behind the standard buffer (Item 1); b) Absorber (Item 1) as a part of shrinking anticlimber system 


\section{Experimental study}

The quasi-static tests have been performed using hydraulic testing machine which can be achieved maximum load of $250 \mathrm{kN}$, Fig. 2(a). All tests were realised on the scaled samples with machine head speed of $1 \mathrm{~mm} / \mathrm{s}$. This value of machine head speed and values very close to this one were used in many previous tests, and in this way is possible to compare and analyse results obtained by these tests with others previous. Results presented in papers [3-8] have shown that scaled samples are suitable for analysing parameters (benefits and weaknesses) of the proposed absorption method and that parameters obtained in this way provide a basis for the final preparation of full size elements of absorber (prototype), which decreases development costs significantly. During axial loading of the absorber assembly, the reaction force at the defined stroke is measured. Deformed foam filled tube is shown in Fig. 2(b). Due to elastic deformations of the tube wall, the end of the tube that is passed cone bushing has a diameter for $\approx 1 \mathrm{~mm}$ higher than the diameter of rest of deformed tube wall. Length of the all samples (seamless tubes) was $110 \mathrm{~mm}$. With the aim to prevent contact between the machine head and the upper end of cone bushing, maximum stroke of $90 \mathrm{~mm}$ was used during tests.

Auxiliary tool used as support of cone bushing during test (Item 1, Fig. 2(a)) was made from steel in grade S355J2G3. Under the support tool is the circular steel plate (Item 2, Fig. 2(a)) for mounting on the base of machine.
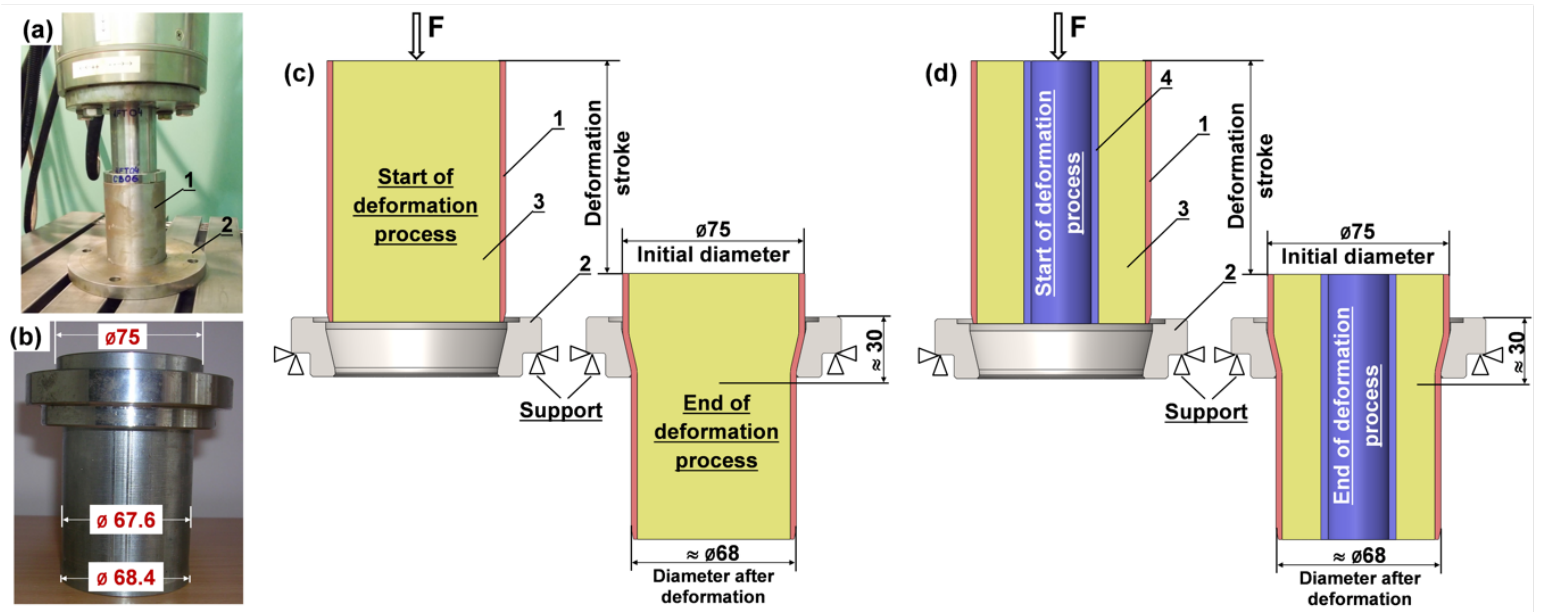

Figure 2. Testing machine and deformed tubes: (a) Assembly mounted on the machine (1-support tool, 2-circular steel plate); (b) Deformed foam-filled tube; (c) Working principle of whole tube volume filled by PU foam (1Seamless steel tube, 2-Steel Cone bushing and 3-Rigid PU foam); (d) Working principle of concentric tube volume filled by PU foam (4-Inner steel tube)

\subsection{Working principles}

Two types of tube arrangement have been analysed: whole filled tube and concentric filled tube. In both configurations, PU foam was used as a filler, Fig. 2(c,d). Analysis and behaviour of the empty tube is considered as a reference. This type of absorber is part of the passive safety system implemented at the end of vehicle structure. When the standard buffer is blocked (fully utilized absorption power of it) the operation of the absorber begins by shrinking the seamless tube filled by rigid PU foam (Item 1 and 3, Fig. 2(c,d)), passing through the cone bushing (Item 2, Fig. 2(c,d)). The initial phase of the deformation process is finishing at the stroke of $\approx 30 \mathrm{~mm}$. At this phase comes to reduction of the foam-filled tube from its initial diameter $(\varnothing 75 \mathrm{~mm})$ to final diameter very close to smaller diameter of the cone bushing ( $\approx \varnothing 67.6 \mathrm{~mm}$ ), Fig. 2(c,d). Once the tube begins to exit the cone bushing, the deformation process is relatively stable and the deformation resistance remains approximately constant to the end of absorption process. Maintaining a smooth and predictable deformation resistance is important for controlling the collision dynamics of vehicles. Using foam filled tube shrinking energy absorber, energy absorption realises in three ways: elastic-plastic deformation of the tube wall, friction between steel absorption elements and compression the rigid PU foam inside the tube. 


\subsection{Preparation of steel samples}

This test procedure requires the specimens configuration with items shown in Fig. 3(a): seamless tubes with outer diameter $\varnothing 75 \mathrm{~mm}$, wall thickness $2.5 \mathrm{~mm}$ and length $110 \mathrm{~mm}$ (Items 1) from structural steel in grade 13CrMo4-5; inner tubes with outer diameter $\varnothing 30$, wall thickness $3.0 \mathrm{~mm}$ and length $\mathrm{L}=110 \mathrm{~mm}$ (Items 2) from low carbon steel in grade S235, and steel covers (Items 3 and 4) from low carbon steel in grade S235. Two types of steel cover were used, one (Item 3, Fig. 3(a)) for whole filled tubes (Fig. 3(b)); and one (Item 4, Fig. 3(a)) for concentric filled tube which contains an inner tube (Fig. 3(c)), where the design of the cover ensures the outer and inner tubes stay concentric during casting process of PU foam.

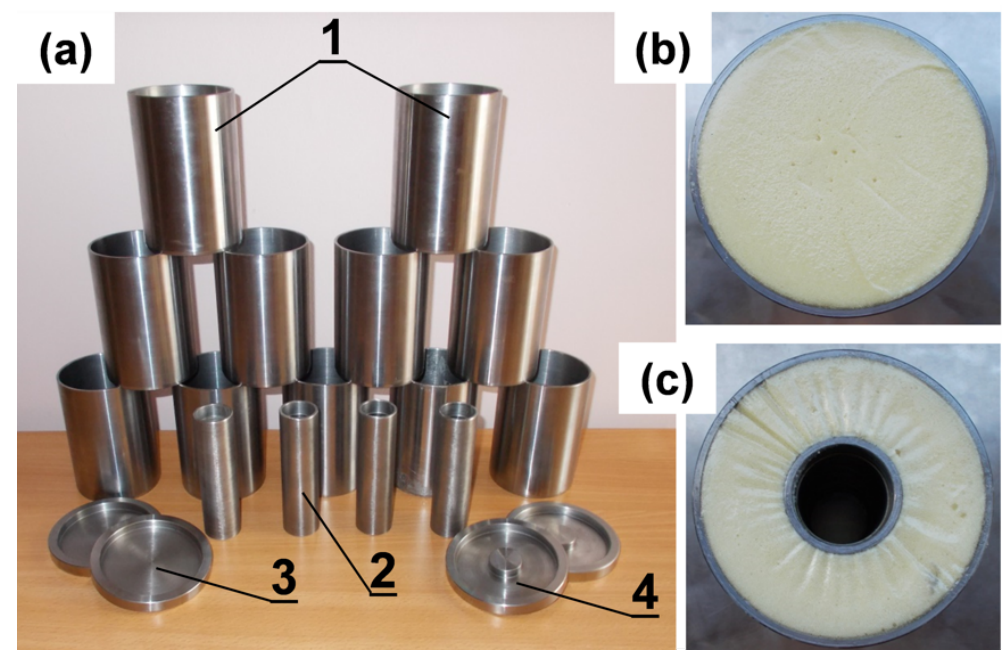

Figure 3. Items and specimens' configurations: (a) Items (1-Seamless steel tube, 2-Inner steel tube, 3-Steel plane covers and 4-Steel concentric covers); (b) Whole filled tube, (c) Concentric filled tube

The mechanical properties of 13CrMo4-5 steel are defined in EN10216-2 [24]. The cone bushing with dimensions $\varnothing 75 / 68 \times 13^{\circ}$ is made from steel in grade C45E: the mechanical properties of it are defined in EN10083-2 [25]. The empty tubes are signed as NET, whole filled tubes are signed as NFT and concentric filled tubes are signed as CFT, while cone bushings are signed as CB. Some physical and mechanical material properties of the tube and cone bushing are presented in Table 1. Values of Yield Strength and Tangent Modulus of steel tube are determined on the base of experimental results of tested specimens.

Table 1 Physical and mechanical material properties of specimens

\begin{tabular}{cccccccc}
\hline Component & $\begin{array}{c}\text { Density } \\
{\left[\mathrm{kg} / \mathrm{m}^{3}\right]}\end{array}$ & $\begin{array}{c}\text { Elastic modulus } \\
{[\mathrm{GPa}]}\end{array}$ & $\begin{array}{c}\text { Tangent } \\
\text { modulus } \\
{[\mathrm{MPa}]}\end{array}$ & $\begin{array}{c}\text { Poisson } \\
\text { ratio }\end{array}$ & $\begin{array}{c}\text { Yield stress } \\
{[\mathrm{MPa}]}\end{array}$ & $\begin{array}{c}\text { Bulk } \\
\text { modulus } \\
{[\mathrm{MPa}]}\end{array}$ & $\begin{array}{c}\text { Shear } \\
\text { modulus } \\
{[\mathrm{MPa}]}\end{array}$ \\
\hline $\begin{array}{c}\text { Seamless } \\
\text { tube } \\
\text { 13CrMo4-5 }\end{array}$ & 7850 & 210 & 785 & 0.3 & 315 & 1666.7 & 769.2 \\
\hline $\begin{array}{c}\text { Cone bushing } \\
\text { C45E }\end{array}$ & 7850 & 210 & - & 0.3 & 490 & - & - \\
\hline
\end{tabular}

\subsection{Polyurethane foam}

Rigid polyurethane (PU) foam was prepared by mixing liquid polyisocyanate with liquid polyol. Precisely defined and measured quantities of the liquids were poured into tubes and mixed together for about 40 seconds. In order to keep the internal pressure produced by chemical process, both ends of the tube were closed tightly by covers (Item 3 and 4 , Fig. 3(a)). During expansion of the polyurethane foam, the chemical process produces high-pressure on the tube wall as well as on the covers of the tube, so it was necessary to perform this process in a strongly controlled environment. Also, during expansion process of the PU foam, push out of compressed air from mould (seamless tube) in the upper 
zone must be provided to uniformly fill the whole volume of the tube. In the case that air stayed under the upper cover, PU foam can't reach surface of it, so a certain of tube volume will not be filled by it. This leads to uncontrolled PU foam density that is undesired in this case. Adequate casting technology is very important in realization of this process. The resultant rigid PU foam density value in the experiment was $170 \mathrm{~kg} / \mathrm{m} 3$ for both types of tubes (NFT and CFT). The PU foam is glued to the tube wall after finished casting process. PU foam specimens with a density of $170 \mathrm{~kg} / \mathrm{m}^{3}$ and dimensions 30x30x30 mm were used for experimental determination of the foam properties (Fig. 4(a)) by uniaxial compression tests in accordance with ISO 844 standard [21]. The axial force was applied on the PU foam samples, using Universal Testing H10K-s machine with maximum of $5 \mathrm{kN}$ load cell (Tinius Olsen, USA). Fig. 4(b) shows engineering compressive stress-strain curve (average values) of the representative samples, obtained by testing. Stressstrain curve shows the predicted conventional hyperelastic curve with 3 distinct regions: elastic region, a middle plateau and a final densification region.
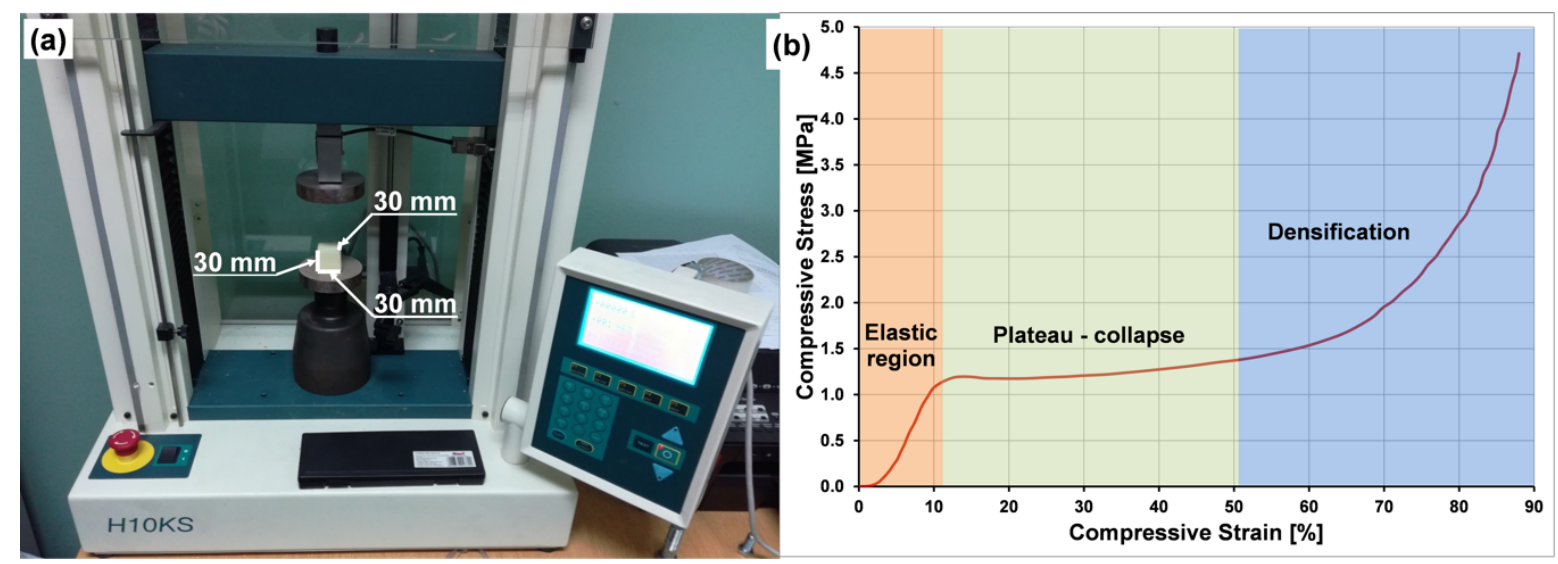

Figure 4. PU testing: (a) Compression testing device and test sample of PU foam; (b) Compressive stress vs. strain diagram

\section{Numerical simulations}

An ANSYS finite elements (FE) model was used to simulate the shrinking process for all three types of samples. The experiments are modelled as a quasi-static process, with axisymmetric geometry. The aim was to validate the FE model to enable further development of absorbers filled with rigid PU foam and the opportunity to analyse in more detail and more clearly the entire process of absorber deformation, reducing thereby the number (including costs) of full-scale tests required for official verification. The following process parameters and behaviour of geometry are essential for further evaluation: the flow of the force-stroke curve, maximum and average values of deformation resistance, and the shape of samples before and after deformation process. The large deformation of the tube observed during tests, together with the complex contact behaviour between the steel elements, indicates the need for nonlinear FE analysis.

\subsection{Finite element model}

The two-dimensional axisymmetric models of the NET, NFT and CFT are presented in Fig. 5(a), with corresponding meshes shown in Fig. 5(b). Discretization of the tube and the cone bushing model with 2D axisymmetric elements by FE method was done. Support tools and other ancillary parts are considered negligible and are not modelled. Mesh density is chosen so the approximate dimensions of elements are $0.5 \times 0.5 \mathrm{~mm}$, which means that in the radial direction of the tube were five elements, Fig. 5(b). The same size of elements was at the contact edge of the cone bushing, to minimise the problem of node penetration and oscillation of contact forces. Element size of PU foam at the interface surface/edge with steel tube is the same like to element size at the steel tube. Bearing in mind that the surfaces of PU foam and steel tube are bonded, after casting process of PU foam, nodes of these two parts are merged. From previous experience, smaller elements increase the total computational time, but will not significantly increase the accuracy of the stress and deformation field [22]. 


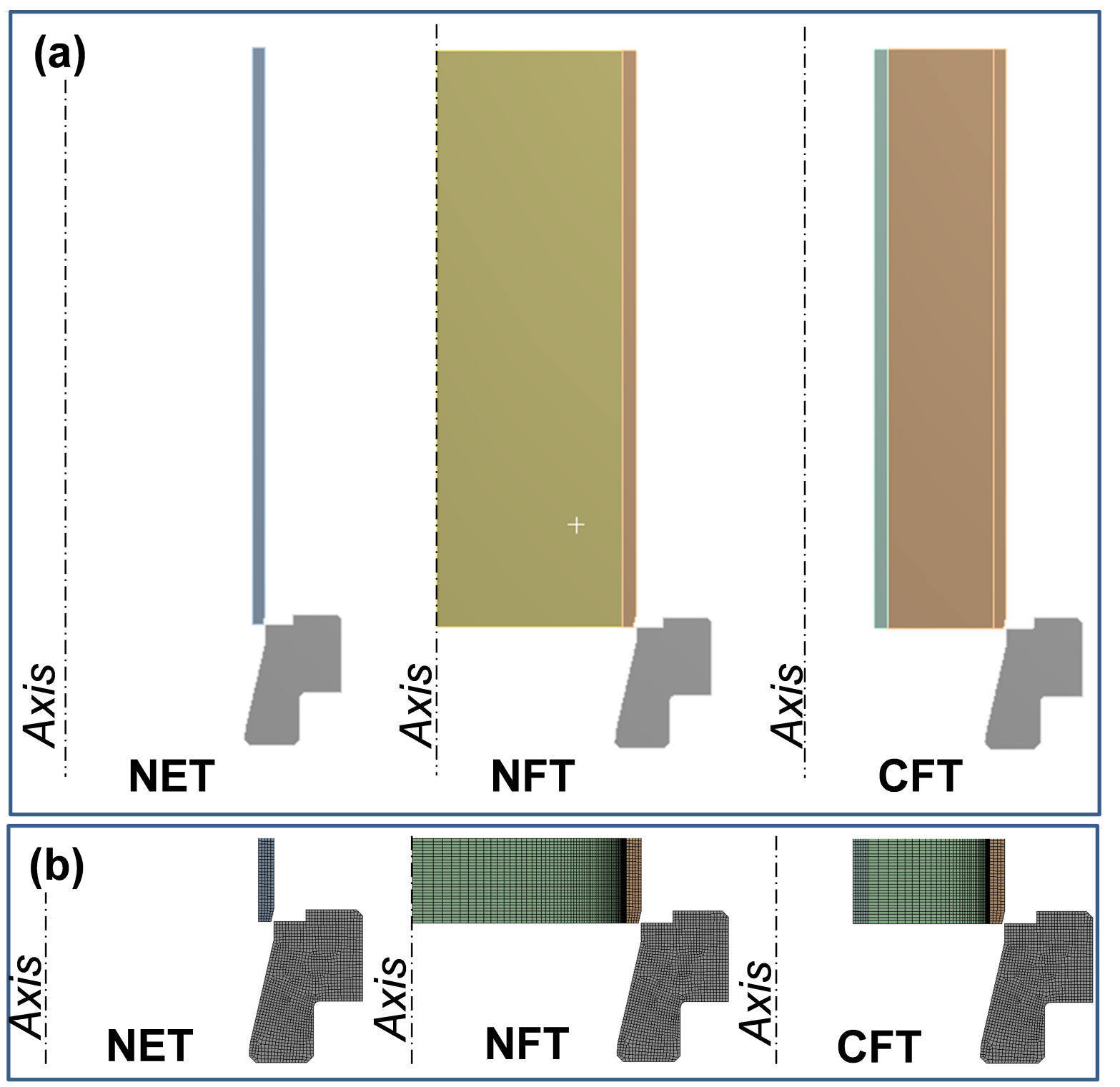

Figure 5. Numerical model of NET, NFT and CFT absorber: (a) 2D plane axisymmetric models of absorber; (b) Meshed numerical models

\subsection{Contact algorithm and boundary conditions}

A realistic simulation of the absorption process has been achieved previously in ANSYS [23] using frictional contact type for sliding surfaces or edges in axisymmetric problems. Energy absorption was directly linked to the friction coefficient; which was adopted from earlier research to be 0.15 [22]. Trim contact algorithm was used with remeshing capabilities, which can reduce the elements for the solver and decrease the calculation time. Formulation method was Augmented Lagrange, which is a penalty-based method. Fixed constraints were applied (zero-displacement boundary conditions) at the point of contact of the cone bushing and the support tool. Implicit solver was engaged, so that the nodes in contact with the machine piston were given a prescribed displacement of $90 \mathrm{~mm}$ with linear growth to the end of movement.

\subsection{Material model of steel tube}

Due to the presence of large deformations, which include the hardening of material through the process of tube deformation, a bilinear material model was chosen for modelling of steel tubes. This material model uses associative 
flow rule with isotropic hardening by the von Mises yield criterion. At the moment when the equivalent (von Mises) stress $\sigma_{e}$ is equal to the current yield stress $\sigma_{c}$, starts yield of material. Since the experimental tests show that hydrostatic stress has negligible effect on plastic deformation, the yield criteria can be written as

$$
F=\sqrt{\frac{3}{2} S_{i j} s_{i j}}-\sigma_{c}=0
$$

where $S_{i j}$ is deviatoric stress tensor. In case for isotropic plasticity, assumed here, current yield stress $\sigma_{c}$ can be calculated from equivalent plastic strain $\hat{\varepsilon}^{p l}$ and experimental determined true stress-strain curve. Current yield stress and equivalent plastic strain are related through plasticity modulus $E_{P}$ in form

$$
\sigma_{c}=E_{P} \hat{\varepsilon}^{p l}
$$

where $E_{p}$ - plastic modulus is calculated by the expression

$$
E_{p}=\frac{E_{t} E}{E-E_{t}}
$$

in which $E$ - Elastic modulus, $E_{t}$ - Tangent modulus. Material properties of the tube and the cone bushing, applied in numerical simulations, were presented in Table 1.

\subsection{Material model of PU foam}

For modelling of PU foam, Ogden Foam material model was chosen with tabular input date of experimental data. The uniaxial compression test is convenient to define the model parameters and is carried out on PU foam as shown in Fig. 4(a). The elastic modulus $\mathrm{E}=66.1 \mathrm{MPa}$ of polyurethane foam is calculated using results obtained by compression test. Poisson's ratio value in elastic and plastic region is adopted to be " 0 ". The initial yield stress in uniaxial compression is the stress value at point where curve passes from elastic region to plateau-collapse region and yield stress during the deformation is determined based on the experimental data corresponding to the curve in plateau region, Fig. 4(b).

\section{Results}

\subsection{Test results -shrinking of the tube}

Force vs stroke $\mathrm{F}(\mathrm{s})$ curve for the compression test for the shrinking of NET is presented in Fig. 6(a). These results present a reference for assessment of absorption properties of the tubes filled by rigid PU foam. Seven samples were tested using shrinking process of NFT, Fig 6(b), and three CFT were deformed, Fig. 6(c). It is characteristic for all three $\mathrm{F}(\mathrm{s})$ diagrams that the force reaches a maximum value at a stroke of about $30 \mathrm{~mm}$ and stays at it (with minor deviation) until finished deformation process. Differences between diagrams show expected higher values of the force by using PU foam filler in comparison to the empty tube. The maximum force value corresponds to the part of the tube wall that has passed cone surface of bushing and have got finally deformed plastically to diameter of $\approx 68 \mathrm{~mm}$. After deformation, the tube length increase in a certain percentage while the change of wall thickness is negligible. In this way the volume of material of the seamless tube remains unchanged. The deviation of steady-state force values, Fig. $6(a, b, c)$ are a consequence of slightly different roughness of the outer tube surface, leading to deviation in the coefficient of friction (quality of machining is very important in manufacturing of this type of elements).

Fig. 6(d) shows characteristic F(s) curves for the shrinking process of all three types of tubes, averaged over the tests for the respective tube configurations. This clearly shows the differences between these three configurations. The clear differences are in the maximum values of the force, as a consequence of rigid PU foam influence. 

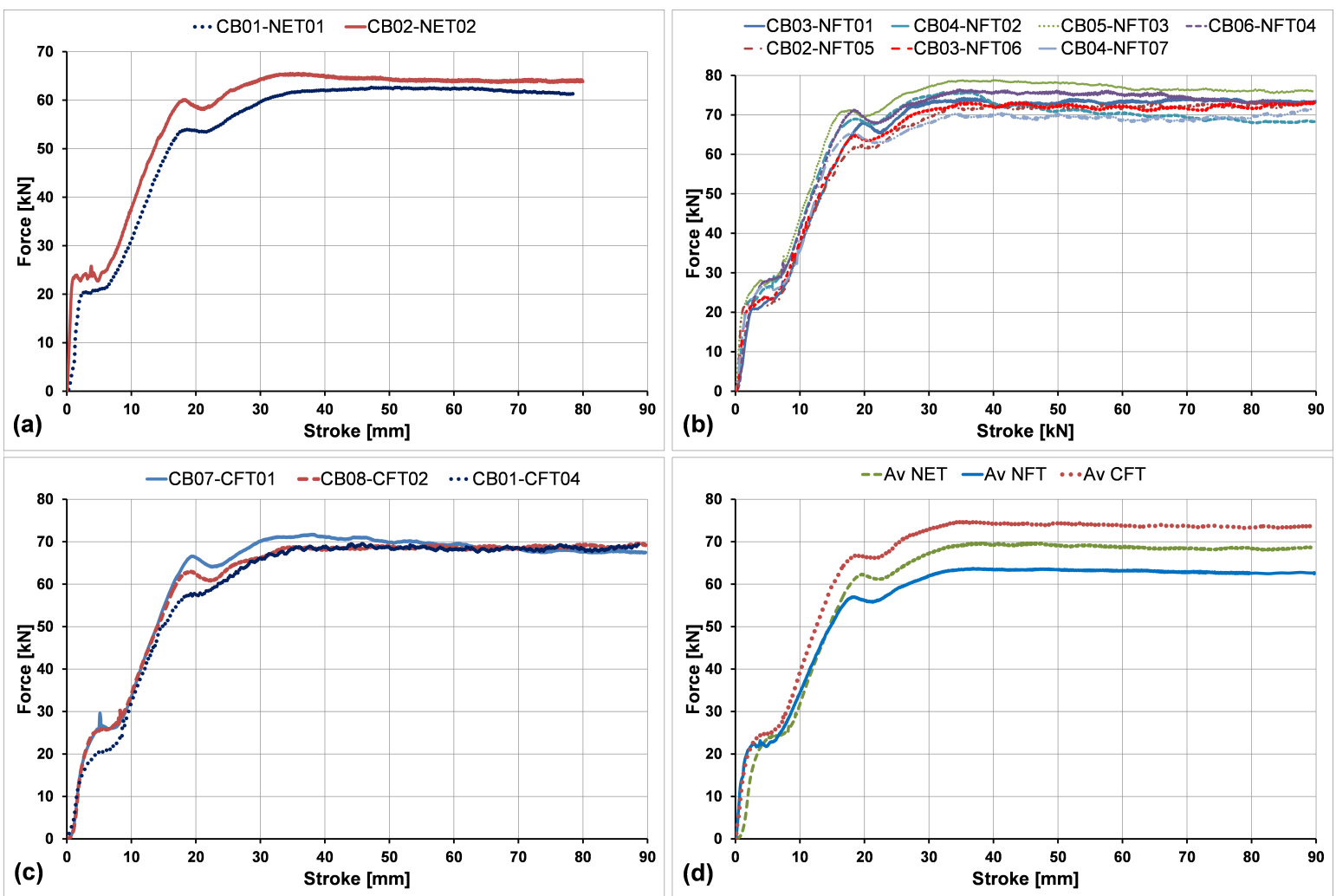

Figure 6. F(s) diagrams - experimental results: (a) NET; (b) NFT; (c) CFT; (d) Average values-comparison

To analyse the properties of shrinking of empty and foam filled steel seamless tubes, the crashworthiness indicators, such as the deformation resistance (F), absorbed energy (EA) and specific absorbed energy (SEA) were used in this study. Absorbed energy (EA) presents the energy absorbed by the tube (NET, NFT and CFT) during the shrinking and can be calculated as:

$$
A E=\int_{0}^{s} F(s) d s
$$

where " $\mathrm{F}$ " is the deformation resistance-force and "s" is the deformation stroke.

Specific absorbed energy (SEA) presents the absorbed energy per unit mass " $m$ " of tested sample and is calculated as:

$$
S A E=A E / m
$$

Table 2 contains main parameters for evaluation of absorption properties.

Parameters presented in Table 2 show differences between EA and SEA. The whole foam-filled tube can absorb about $18 \%$ higher collision energy in comparison to the empty one, while the concentric foam-filled tube gives about $9 \%$ higher. A larger mass of concentric type of absorber as a consequence of the inner steel tube causes decrease of values of specific energy absorbed for about $45 \%$ in comparison with empty and whole filled tube. One more advantage of whole filled tube is simpler construction as well as easier casting of PU foam. By analysing these parameters it is clear that the tube whose entire volume is filled by PU foam is more suitable for use in energy absorption in comparison to concentric foam filled tube. Whole filled tubes will be used for further analysis. 
Table 2 Main parameters - experimental investigations

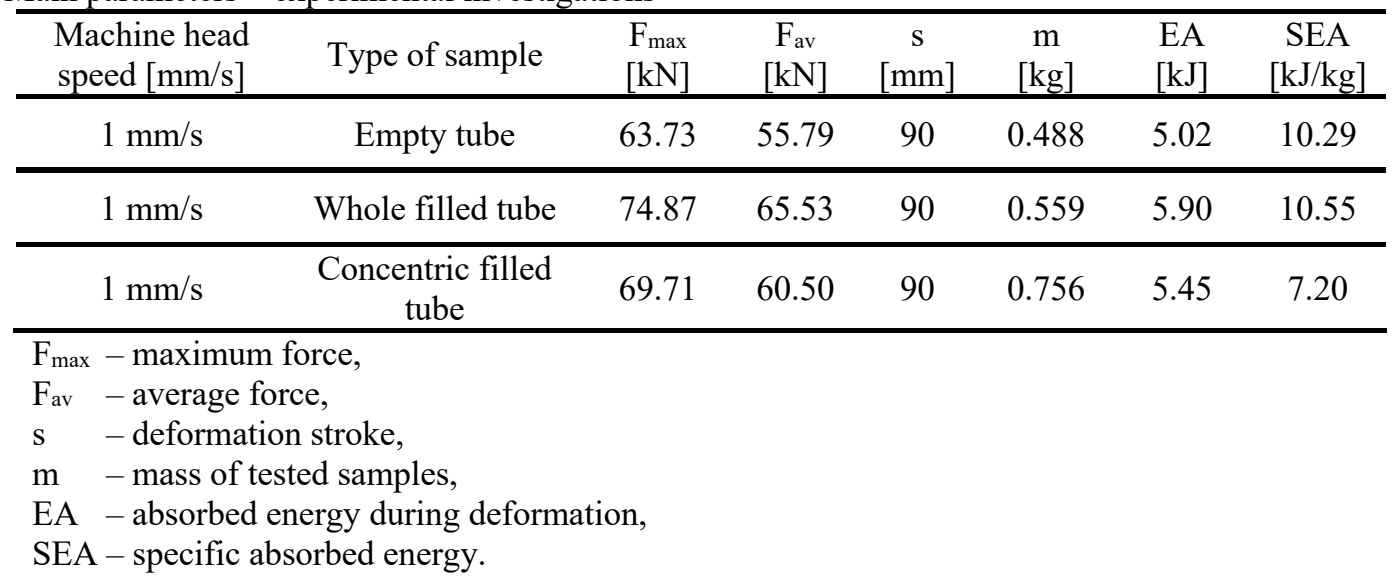

\subsection{Numerical simulations results - shrinking of the tube}

The F(s) diagram obtained by FE analyses using ANSYS Workbench is presented in Fig. 7(a). Numerical simulations were performed for all three tube configurations: NET, NFT and CFT (see Fig. 5). The F(s) curves have the same characteristics as the corresponding test results: a gradual increase of force to the maximum value, when the force stays on this value until deformation process is finished. It is clear that deformation process is divided in three phases for all tested configurations. First phase is on the stroke of $\approx 8 \mathrm{~mm}$. In this phase, started surface contact between chamfered outside edge of the tube and fixed cone bushing leads to sharp force increase until the moment when force start to decrease as a consequence of bending of end of the tube to the axis of it (surface contact transfer to line contact). Second phase starts with second wave of force increase at the moment when again is formed surface contact between the tube and cone bushing. Further progression of the defined stroke leads to increase of contact surface until the end of the tube starts coming out of the cone bushing. At this moment starts third phase. Third phase characterizes slightly increase of force to the end of relaxation of the tube end which passed cone bushing. After that force has an approximately constant value. For all three configurations first and second phase are the similar (energy is wasted on inside deformation of steel tube). In the third phase, when the deformation process of steel tube passed to stable phase, increase of the force is results of influence of inside energy of PU foam. Higher volume of PU foam leads to higher value of the force, so configuration without inner tube is more acceptable.

Comparative $\mathrm{F}(\mathrm{s})$ curves of the three tube configurations, obtained through tests and ANSYS simulations, are shown in Fig. 7(b). Numerical and test results match closely for all three configurations. For the NET and CFT, the maximum deviations are less than 3\%, while for the NFT deviations are less than 5\%. Fig. 7(e) presents the von Mises stress distribution of the whole foam-filled tube obtained by FE simulations using ANSYS Workbench. This figure shows that the shape of deformation of the whole foam-filled tube obtained by numerical simulations are in a good correlation with experimental one (see Fig. 2(b)). Also, these two figures show very close values of diameters (at two positions of tube wall passed through cone bushing) measured on tested sample and deformed numerical model (Fig. 7(e)). Maximal value of the stress is on the tube part which passed through cone bushing. This state confirms that the end of tube wall has a certain unloading after passed cone bushing, as a consequence of elastic deformation (spring back effect). The close match of results validates the developed FE model for further research work with this type of elements and for additional research analyses as well as for pre-dimensioning of full-scale elements. 

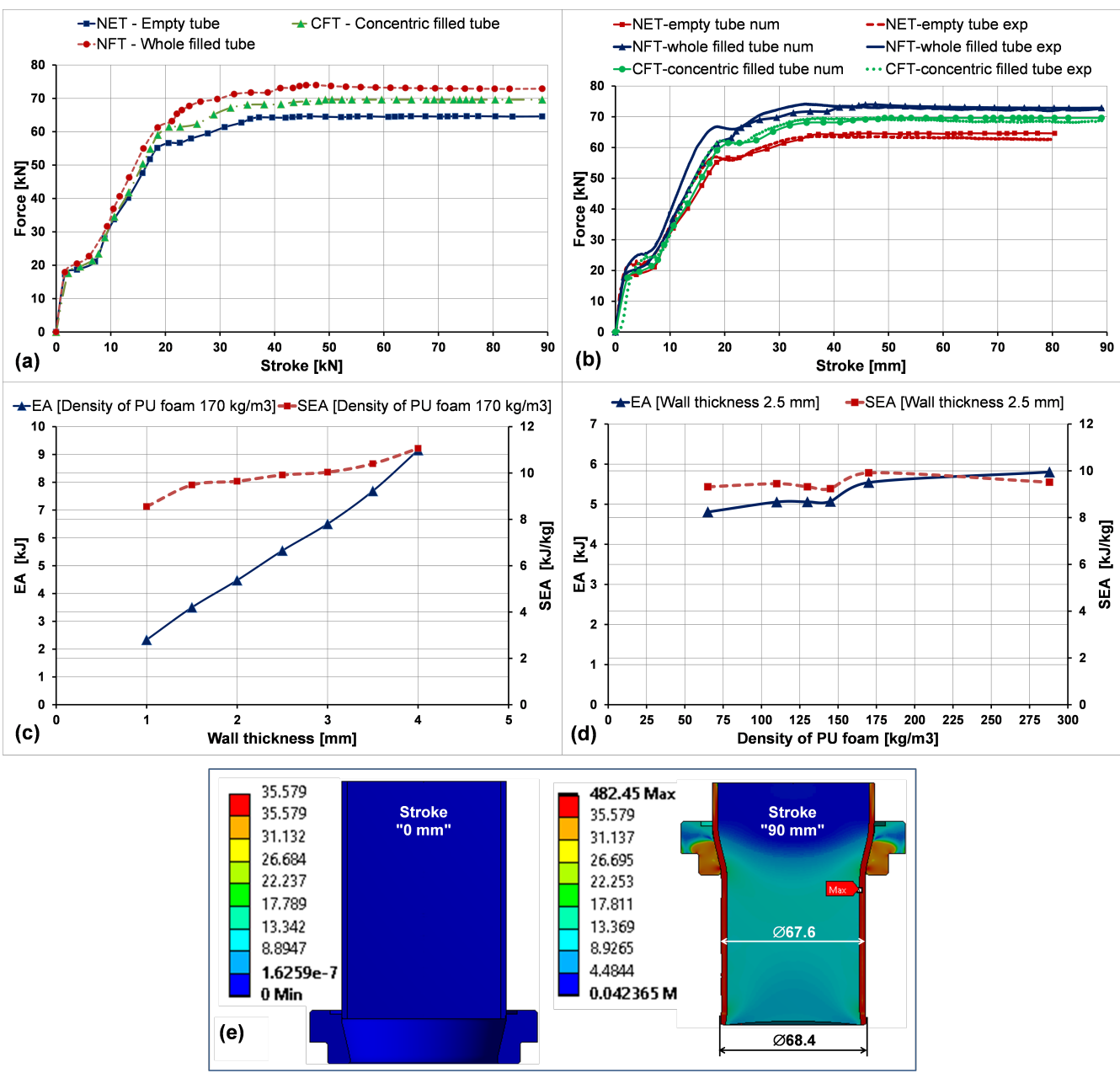

Figure 7. Numerical simulations of whole filled tubes: (a) F(s) diagram - ANSYS Workbench; (b) F(s) diagram Validation of numerical model; (c) EA and SEA vs. wall thickness; (d) EA and SEA vs. density; (e) von Mises Stress distributions [MPa] of NFT specimen

\subsection{Influence of tube wall thickness and density of $P U$ foam on the absorption characteristics}

One of benefits of development and validation of numerical model is the possibility of using it to study different parameters which can make simplify further investigations of this and similar types of elements. The density of PU foam and tube wall thickness have a main impact on absorption power, next to the friction which will not be consider in this paper. These analyses were done for whole filled tube absorber. Table 3 contains key parameters obtained by numerical simulations of shrinking process using different values of tube wall thickness and constant value of density of PU foam $\left(170 \mathrm{~kg} / \mathrm{m}^{3}\right)$ as well as different values of density of PU foam and constant value of wall thickness of 2.5 $\mathrm{mm}$. Mechanical properties in FE analyses, for different density of PU foam, were taken from the research papers [2831 ] and implemented in the developed numerical model.

Fig. 7(c) shows curves of EA/SEA vs. wall thickness, for seven different values of wall thickness, obtained through of numerical simulations. This diagram shows that increasing of wall thickness causes a significant increase of EA, while the SEA slightly increases. This was expected because thicker wall leads to significant increase of deformation resistance as well as mass of elements. Next to the energy absorbed, having in mind that the design of the collision energy absorber is conditioned by the Railway standards, a great care should be addressed to the values of the deformation resistance. 
Table 3 Key parameters - different values of wall thickness and PU density

\begin{tabular}{cccccccc}
\hline $\begin{array}{c}\text { Wall thickness } \\
{[\mathrm{mm}]}\end{array}$ & $\begin{array}{c}\text { Density } \\
{\left[\mathrm{kg} / \mathrm{m}^{3}\right]}\end{array}$ & $\begin{array}{c}\mathrm{F}_{\max } \\
{[\mathrm{kN}]}\end{array}$ & $\begin{array}{c}\mathrm{F}_{\mathrm{av}} \\
{[\mathrm{kN}]}\end{array}$ & $\begin{array}{c}\mathrm{h} \\
{[\mathrm{mm}]}\end{array}$ & $\begin{array}{c}\mathrm{m} \\
{[\mathrm{kg}]}\end{array}$ & $\begin{array}{c}\mathrm{AE} \\
{[\mathrm{kJ}]}\end{array}$ & $\begin{array}{c}\mathrm{SAE} \\
{[\mathrm{kJ} / \mathrm{kg}]}\end{array}$ \\
\hline 1 & 170 & 29.87 & 25.94 & 90 & 0.273 & 2.33 & 8.55 \\
\hline 1.5 & 170 & 43.98 & 38.88 & 90 & 0.369 & 3.50 & 9.48 \\
\hline 2.0 & 170 & 57.93 & 49.72 & 90 & 0.464 & 4.47 & 9.64 \\
\hline 2.5 & 170 & 73.96 & 61.58 & 90 & 0.559 & 5.54 & 9.91 \\
\hline 3.0 & 170 & 90.43 & 72.25 & 90 & 0.648 & 6.50 & 10.03 \\
\hline 3.5 & 170 & 106.27 & 85.40 & 90 & 0.739 & 7.69 & 10.40 \\
\hline 4.0 & 170 & 127.82 & 101.74 & 90 & 0.828 & 9.16 & 11.06 \\
\hline 2.5 & 65 & 64.24 & 53.41 & 90 & 0.516 & 4.81 & 9.32 \\
\hline 2.5 & 110 & 67.19 & 56.18 & 90 & 0.535 & 5.06 & 9.45 \\
\hline 2.5 & 130 & 68.78 & 56.21 & 90 & 0.543 & 5.06 & 9.32 \\
\hline 2.5 & 145 & 69.39 & 56.35 & 90 & 0.549 & 5.07 & 9.24 \\
\hline 2.5 & 170 & 73.96 & 61.58 & 90 & 0.559 & 5.54 & 9.91 \\
\hline 2.5 & 288 & 75.62 & 64.48 & 90 & 0.610 & 5.80 & 9.51 \\
\hline
\end{tabular}

Increasing of wall thickness from $1.0 \mathrm{~mm}$ up to $2.5 \mathrm{~mm}$ gives up to 2.5 times larger values of the force, while increase from $2.5 \mathrm{~mm}$ up to $4 \mathrm{~mm}$ gives up to 1.8 times larger values of the force (Table 3). High values of deformation resistance result in increased transfer of collision force to the wagon structure which can induce uncontrolled deformation of it. The initial value of deformation resistance should therefore be aligned with maximal allowed force in the standard buffer or automatic coupler in normal exploitation $[1,2]$.

Fig. 7(d) shows curves of EA/SEA vs. density of PU foam for six different values of density obtained through of numerical simulations. As expected, influence of density of PU foam is smaller in comparison with influence of tube wall thickness. However, this fact not gives advantage of changing of wall thickness as a solution to increase of absorption power. This diagram shows that increasing of density causes a slight increase of EA in a whole region, while the SEA slightly increases up to density of $170 \mathrm{~kg} / \mathrm{m}^{3}$ and then starts to decrease. Mechanical properties for different density of PU foam are taken from the papers [28-31]. Possible of using different methods and certain errors during determination of characteristics of PU foam can be reason of slightly decreasing of EA and SEA from density 100 until $150 \mathrm{~kg} / \mathrm{m}^{3}$. Also, deformation resistance as well as mass of elements have slight increasing in comparison with previous analysis. Increasing of density from 65 up to $288 \mathrm{~kg} / \mathrm{m}^{3}$ gives for about $16 \%$ larger values of the force (Table 3).

\subsection{Implications of foam-filled absorber in vehicle collision}

Collision energy absorber has a role to absorb a certain amount of collision kinetic energy and in this way to reduce forces that will transfer to the passenger zone of bearing structure. In this way passive safety elements protect passengers, goods and wagon from undesired consequences. Rigid PU foam as filler will enable fine tuning of desired parameters (deformation resistance-force) without a large increase of mass of absorber assembly. Four shrinking foamfilled tube absorbers for a passenger wagon will have mass of about $500 \mathrm{~kg}$. Passive safety elements are a critical feature of wagons, with impact energy absorbers providing a steady, controlled resistance over a defined stroke. The results presented here show that resistance and energy absorbed increase linearly with wall thickness, but thick walls limit the potential for second-stage absorber features to increase the stroke and thus the total energy absorbed. The 
results show also that the foam's absorbent properties are not being fully exploited; one potential design improvement would be to use a sharp tool to pierce the foam-filled tube along its axis, to radially expand and crush the foam as it passes through the shrinker.

\section{Conclusions}

With the ever greater demands on railways for passenger and freight transport, there is increasing need for railway vehicles to be safe and reliable, ready for high-speed operation and equipped with modern safety systems. Most railways have a large number of older passenger and freight wagons as well as locomotives; whose have no installed crush elements that are over needed. For these railway vehicles it is necessary to develop crush elements that can be easy fit into the existing state of the structure, and can meet absorption requirements. These improvements are possible to accomplish during modernisation of existing vehicles.

One of the possible solutions is to use a steel tube with rigid PU foam inside it. This choice was tested and analysed in this work. This type of crash element absorbs energy by shrinking-extruding of a seamless tube filled with rigid PU foam passing through special cone bushing. Two configurations were investigated: whole and concentric tube volume filled by PU foam. A larger mass and less absorption power put foam filled concentric tube behind the whole filled tube absorber which is more suitable for use.

Research presented in this paper shows that rigid PU foam inside the tube can increase absorption power. Also, it is possible to fine-tune the value of deformation resistance by changing density of PU foam or tube wall thickness. Analyses show that increase of wall thickness gives significant increase of force and mass of the assembly. On the other side, increase of density of PU foam gives gradual increase of force and less mass of the assembly. Based on these conclusions and request that absorber would have compact dimensions and as lower as possible mass, it is more suitable to change absorption power by changing PU foam density than wall thickness.

The shrinking foam filled tube absorber is intended to be used behind the standard buffer or the anticlimber devices. Both solutions of installation require exactly defined value of deformation resistance (force) when the absorber should start to deform and absorb energy. This moment is immediately after fully used absorption power of the standard buffer (passenger and freight wagons) or coupler (DMU and EMU). Defining the optimal dimensions of the tube and influence of rigid PU foam on the absorption power as well as determining of value of the force (deformation resistance) at which the absorber starts process of energy absorption can be realized by using developed numerical model. Further work includes testing and analysis of full scale model of foam filled tube and its dynamic behaviour, plans to be present in another paper on the theme.

Funding: The research work is funded by the Ministry of Education, Science and Technological Development of Republic of Serbia, Projects TR35045, TR35006 and TR35031 and 451-03-68/2020-14/200378. 


\section{References}

1. EN 15227:2008. Railway applications - Crashworthiness requirements for railway vehicle body.

2. EN 12663-1:2010. Railway applications - Structural requirements of railway vehicle bodies - Part 1: Locomotives and passenger rolling stock (and alternative method for freight wagons).

3. Simić G, Lučanin V, Tanasković J, et al. Experimental research of characteristics of shock absorbers of impact energy of passenger coaches, Exp Tech 2009; 33: 29-35.

4. Lučanin V, Tanasković J, Milković D, et al. Experimental Research of the Tube Absorbers of Kinetic Energy During Collision, FME Transactions 2007; 35: 201-204.

5. Tanasković J, Milković D, Lučanin V, et al. Experimental and numerical determination of tube collision energy apsorbers characteristics, FME Transactions 2012; 40: 11-16.

6. Tanaskovic J, Lučanin V, Milković D, et al. Experimental Research of Characteristics of Modified Tube Absorbers of Kinetic Collision Energy of Passenger Coaches, Exp Tech 2014; 38: 37-44.

7. Tanaskovic J, Milkovic D, Lucanin V, et al. Experimental investigations of the extrusion-splitting tube collision energy absorber, Thin Wall Struct 2015; 86: 142-147.

8. Tanaskovic J, Milkovic D, Lucanin V, et al. Experimental and numerical analysis of the characteristics of combined collision energy absorbers, FACTA UNIVERSITATIS Series: Mechanical Engineering 2012; 10: 125 - 136.

9. Reddy Y and Wall J. Axial compression of foam-filled thin-walled circular tubes, Int. J. Impact Engng 1988; 7 : 151-166.

10. Goel M. Deformation, energy absorption and crushing behavior of single, double-and multi-wall foam filled square and circular tubes, Thin Wall Struct 2015; 90: 1-11.

11. Rezvani $\mathrm{M}$ and Jahan A. Effect of initiator, design, and material on crashworthiness performance of thin-walled cylindrical tubes: A primary multi-criteria analysis in lightweight design, Thin Wall Struct 2015: 96: 169-182.

12. Hussein R, Ruan D, Lu G, et al. Crushing response of square aluminium tubes filled with polyurethane foam and aluminium honeycomb, Thin Wall Struct 2017: 110: 140-154.

13. Elahi S, Rouzegar J, Niknejad A, et al. Theoretical study of absorbed energy by empty and foam-filled composite tubes under lateral compression, Thin Wall Struct 2017: 114: 1-10.

14. Mirfendereski L, Salimi M and Ziaei-Rad S. Parametric study and numerical analysis of empty and foam-filled thin-walled tubes under static and dynamic loadings, Int J Mech Sci 2008: 50: 1042-1057.

15. Karagiozova D, Shu W, Lu G, et al. On the energy absorption of tube reinforced foam materials under quasi-static and dynamic compression, Int J Mech Sci 2016; 105: 102-116.

16. Jafarian B, Rezvani M. An experimental investigation on energy absorption of thin-walled bitubal structures by inversion and axial collapse, Int J Mech Sci 2017; 126: 270-280.

17. Dirgantara T, Jusuf A, Kurniati A, et al. Crashworthiness analysis of foam-filled square column considering strain rate effect of the foam, Thin Wall Struct 2017; 129: 365-380.

18. Darvizeh A, Meshkinzar A, Alitavoli M, et al. Low velocity impact of empty and foam filled circumferentially grooved thick-walled circular tubes, Thin Wall Struct 2017; 110: 97-106.

19. Yalçı $\mathrm{M}$ and Genel K. On the axial deformation characteristic of PVC foam-filled circular aluminium tube: Effect of radially-graded foam filling, Thin Wall Struct 2019; 144: 106335.

20. Wang Y, Pokharel R, Lu J, et al. Experimental, numerical, and analytical studies on polyurethane foam-filled energy absorption connectors under quasi-static loading, Thin Wall Struct 2019; 144: 106257.

21. ISO 844:2007, Rigid cellular plastics - Determination of compression properties, Fifth edition.

22. Tanaskovic J, Franklin F, Dišić A, et al. Numerical validation of the combined extrusion-splitting process of energy absorption through experimental study, Exp Tech 2017; 41: 421-431.

23. ANSYS $2019 \mathrm{r}$, software package - academic

24. EN 10216-2:2013. Seamless steel tubes for pressure purposes - Technical delivery conditions - Part 2: Non-alloy and alloy steel tubes with specified elevated temperature properties.

25. EN 10083-2:2006. Steels for quenching and tempering - Part 2: Technical delivery conditions for non-alloy steels.

26. Moreno C, Reid S and Williams T. Experimental and numerical assessment of oblique loading quasi-static testing of railway anticlimbers, Proc IMechE Part F: J Rail and Rapid Transit 2020; 0 (0): 1-12 https://doi.org/10.1177/0954409720908992

27. Kim S, Shin H, Rhim S, et al. Calibration of hyperelastic and hyperfoam constitutive models for an indentation event of rigid polyurethane foam, Composite Part B 2019; 163: 297-302. 
28. Zarei R and Ghamarian A. Experimental and Numerical Crashworthiness Investigation of Empty and Foam-Filled Thin-Walled Tubes with Shallow Spherical Caps, Exp Mech 2014; 54: 115-126.

29. Park K, Kim H, Her N, et al. Variation of Mechanical Characteristics of Polyurethane Foam: Effect of Test Method; Materials 2019; 12: 2672.

30. Azarakhsh S, Rahi A, Ghamarian A, et al. Axial crushing analysis of empty and foam-filled brass bitubular cylinder tubes, Thin Wall Struct 2015; 95: 60-72.

31. Mane J, Chandra S, Sharma S, et al. Mechanical Property Evaluation of Polyurethane Foam under Quasi-static and Dynamic Strain Rates- An Experimental Study, Procedia Engineering 2017; 173: 726-731.

\section{List of Figures}

Figure 1. Possible mounting methods: (a) Absorber (Item 2) behind the standard buffer (Item 1); b) Absorber (Item 1) as a part of shrinking anticlimber system

Figure 2. Testing machine and deformed tubes: (a) Assembly mounted on the machine (1-support tool, 2-circular steel plate); (b) Deformed foam-filled tube; (c) Working principle of whole tube volume filled by PU foam (1-Seamless steel tube, 2-Steel Cone bushing and 3-Rigid PU foam); (d) Working principle of concentric tube volume filled by PU foam (4-Inner steel tube)

Figure 3. Items and specimens' configurations: (a) Items (1-Seamless steel tube, 2-Inner steel tube, 3-Steel plane covers and 4-Steel concentric covers); (b) Whole filled tube, (c) Concentric filled tube

Figure 4. PU testing: (a) Compression testing device and test sample of PU foam; (b) Compressive stress vs. strain diagram

Figure 5. Numerical model of NET, NFT and CFT absorber: (a) 2D plane axisymmetric models of absorber; (b) Meshed numerical models

Figure 6. F(s) diagrams: (a) NET; (b) NFT; (c) CFT; (d) Average values comparation

Figure 7. (a) F(s) diagram - ANSYS Workbench; (b) F(s) diagram - Validation of numerical model; (c) EA and SEA vs. wall thickness; (d) EA and SEA vs. density; (e) von Mises Stress distributions [MPa] of NFT specimen

\section{List of Tables}

Table 1 Physical and mechanical material properties of specimens

Table 2 Main parameters - experimental investigations

Table 3 Key parameters - different values of wall thickness and PU density 